\title{
COMPARATIVE ACCURACY OF STANDARD ECG ALGORITHMS FOR THE DIAGNOSIS OF BROAD COMPLEX TACHYCARDIA IN CASES OF ATRIAL FLUTTER WITH 1:1 CONDUCTION
}

V N Nathwani, K W Wong, M J Jones, C H Hayes, K R Rajappan, T B Betts, Y B Bashir Oxford University Hospitals

doi:10.1136/heartjnl-2013-304019.67

Background Atrial flutter with 1:1 conduction often presents with broad complex tachycardia (BCT), commonly misdiagnosed as ventricular tachycardia (VT). The utility of standard ECG algorithms for classification of BCT has not been evaluated in this subgroup.

Methods 25 patients had confirmed atrial flutter with 1:1 conduction presenting as BCT. 12/25 (48\%) were taking class I agents. The 12-lead ECGs were independently classified as 'SVT' or 'VT' by two blinded electrophysiologists, using five established BCT algorithms: Wellens et al 1978; Brugada et al 1991; Griffith et al 1994; Vereckei et al 2008; Pava et al 2010.

Results Mean ventricular rate $219 \pm 34 \mathrm{bpm}, \mathrm{LBBB}$ pattern in 19/25 (76\%), ORS duration $>140 \mathrm{~ms}$ in $20 / 25(80 \%)$ and left axis deviation in $14 / 25$ (56\%) patients. Possible (but not definite) VA-dissociation was noted in 10 cases (40\%). There was close interobserver agreement. The results of the ECG analysis were:

There was a high rate of mis-classification as VT by all algorithms except Pava ( $\mathrm{R}$ wave peak time at DII or RWPT) which correctly identified the origin in $76 \%$ of cases.

Conclusions Atrial flutter presenting with 1:1 conduction and BCT is often mistaken for VT and 'pseudo-VA dissociation' is relatively common. Standard ECG algorithms are relatively inaccurate in this subgroup, except for RWPT which identified the supraventricular origin in $76 \%$ of cases. A high level of vigilance is necessary to make the correct diagnosis

Table 1

\begin{tabular}{lrcc}
\hline & SVT & VT & Accuracy \\
\hline Wellens 1978 & 4 & 21 & $16 \%$ \\
Brugada 1991 & 5 & 20 & $25 \%$ \\
Griffith 1994 & 14 & 11 & $56 \%$ \\
Vereckei 2008 & 7 & 18 & $28 \%$ \\
Pava 2010 & 19 & 6 & $76 \%$ \\
\hline
\end{tabular}

